\title{
Percutaneous Pedicle Screw Fixation in Thoracolumbar Fractures: Comparison of Results According to Implant Removal Time
}

\author{
Ho-Seok Oh, MD, Hyoung-Yeon Seo, MD \\ Department of Orthopedic Surgery, Chonnam National University Hospital, Gwangju, Korea
}

\begin{abstract}
Background: The purpose of this study was to determine whether restoration of range of motion (ROM) could be achieved by implant removal after natural bone healing and consolidation of fractured vertebrae and examine whether early removal of the implant could maximize restoration of ROM.

Methods: This study included 30 cases of thoracolumbar fractures without neurological deficit requiring surgery (nine cases of flexion-distraction injuries and 21 cases of burst fractures). Percutaneous pedicle screw fixation (PPSF) was performed at the fractured vertebrae and one level above and one level below the fracture level. Pedicle screws were removed at an average of 12 months after surgery upon healing of fractured vertebrae. The following radiological and clinical findings were evaluated: restoration of anterior vertebral height ratio (AVHR), Cobb angle (CA), ROM, and complications. Sixteen patients who were checked for ROM were divided into two groups based on the time of implant removal: nine patients within 12 months and seven patients after 12 months. Restoration of vertebral height loss and ROM were compared between the two groups.

Results: At the final follow-up, significant pain relief and restoration of AVHR and CA were achieved in patients who underwent PPSF. Patients who had implant removed within 12 months after surgery had better ROM recovery than those who had implant removed after 12 months postoperatively. There were no significant differences in AVHR and CA between the two groups.

Conclusions: PPSF followed by implant removal after healing of fractured body appears to be effective in achieving restoration of ROM. In our study, early removal of implant within 12 months after surgery was associated with better achievement of ROM than removal after 12 months. In addition, there were no significant differences in restoration of vertebral height between the two groups.
\end{abstract}

Keywords: Spinal fractures, Pedicle screw, Kyphosis, Range of motion, Device removal

Conventional posterior short-segment pedicle screw fixation was widely accepted for the treatment of thoracolumbar fractures in the past. ${ }^{1-3)}$ However, patients who un-

Received January 9, 2019; Revised March 4, 2019; Accepted March 11, 2019

Correspondence to: Hyoung-Yeon Seo, MD

Department of Orthopedic Surgery, Chonnam National University Hospital, Chonnam National University Medical School, 42 Jebong-ro, Dong-gu, Gwangju 61469, Korea

Tel: +82-62-220-6336, Fax: +82-62-225-7794

E-mail: hyse02001@daum.net derwent the conventional operation suffered from many sequelae such as muscular atrophy, injury, and severe pain due to soft tissue stripping. Recently, many studies have recommended percutaneous pedicle screw fixation (PPSF) as an alternative treatment for thoracolumbar fractures. It results in minimal soft tissue injury and perioperative morbidity. ${ }^{4-6)}$

Some authors have shown that PPSF has many advantages including decreased sequelae, compared to the conventional posterior short-segment pedicle screw fixation. ${ }^{7)}$ However, few studies have investigated clinical 
Oh and Seo. Percutaneous Pedicle Screw Fixation in Thoracolumbar Fracture

Clinics in Orthopedic Surgery • Vol. 11, No. 3, $2019 \bullet$ www.ecios.org

outcomes, maintenance of anterior vertebral height, and range of motion (ROM) after implant removal in patients who underwent the short-segment fixation using percutaneous screws. Early removal of the implant leads to correction loss in vertebral height and Cobb angle (CA), whereas late removal of the implant causes stiffness during intervertebral movements. Therefore, the objective of this study was to investigate whether implant removal after vertebral fracture consolidation could preserve ROM. In addition, we sought to determine the proper time for implant removal to maximize restoration of ROM. ${ }^{8,9)}$

This study hypothesized that restoration of ROM could be achieved by implant removal performed after natural bone healing and consolidation of fractured vertebrae and that early removal of the implant could maximize restoration of ROM.

\section{METHODS}

\section{Patients and Study Design}

This study enrolled 30 patients (16 males and 14 females; between 16 and 73 years of age) whose thoracolumbar burst fracture or flexion-distraction injury was diagnosed between March 2011 and October 2017 at Chonnam National University Hospital. We checked magnetic resonance imaging (MRI) scans of all patients to evaluate the thoracolumbar injury classification and severity score (TLICS) and posterior ligamentous complex injury. Patients with TLICS of 4 or more were treated surgically with PPSF. They underwent implant removal at an average of 12.8 months after fixation. The average follow-up was 18.3 months after surgery, and 5.5 months after implant removal. Their injuries were caused by traffic accidents (10 patients), falling (17 patients), direct injury (two patients), and slipping down (one patient). The fractured vertebra was T11 in one patient, T12 in two patients, L1 in 10 patients, L2 in eight patients, L3 in five patients, and L4 in four patients (Table 1). To investigate whether the time of implant removal influences anterior vertebral height ratio (AVHR) and ROM, 16 patients who were checked for ROM were divided into two groups based on the time of implant removal. Group A consisted of nine patients whose implant was removed within 12 months after surgery while group B consisted of seven patients whose implant was removed at more than 12 months after surgery (Table 2).

We conducted this study in compliance with the principles of the Declaration of Helsinki. The protocol of this study was reviewed and approved by the Institutional Review Board of Chonnam National University Hospital
(IRB No. CNUH-2019-004). Written informed consents were obtained.

\section{Surgical Procedures}

All procedures were performed under general anesthesia in prone position. The fracture site was confirmed by fluoroscopy, and the entry point was set. An approximately $2-\mathrm{cm}$ incision was done, and a pedicle pilot hole was made using a guide wire and a dilator. Pedicle screws were inserted into the fractured vertebra and one level above and one level below the fractured level. After pedicle screw insertion, a rod was also inserted using a special placement system.

\section{Clinical and Radiological Evaluations}

Functional outcome was assessed by Oswestry disability index (ODI). Back pain was quantified using a visual analog scale (VAS). Radiological assessment was performed after implant removal regarding AVHR, sagittal CA, and intersegmental $\mathrm{ROM}$ with supine anteroposterior lateral and flexion-extension roentgenograms. Supine radio-

\section{Table 1. Patient Demographics}

\begin{tabular}{|lc}
\hline \multicolumn{1}{|c}{ Variable } & Value \\
\hline No. of patients & 30 \\
\hline Age (yr) & $41.4 \pm 16.0(16-73)$ \\
\hline Sex (male:female) & $16: 14$ \\
\hline Fracture type & \\
\hline Burst fracture & 21 \\
\hline Flexion-distraction injury & 9 \\
\hline Vertebral level fractured & \\
\hline T11 & 1 \\
\hline T12 & 2 \\
\hline L1 & 10 \\
\hline L2 & 8 \\
\hline L3 & 5 \\
\hline L4 & 4 \\
\hline TLICS & $5.6 \pm 2.2$ \\
\hline Follow-up period (mo) & $18.3 \pm 21.4$ \\
\hline Implant removal (mo) & $12.8 \pm 7.8$ \\
\hline
\end{tabular}

Values are presented as mean \pm SD (range) or mean \pm SD . TLICS: thoracolumbar injury classification and severity score, SD: standard deviation. 
Oh and Seo. Percutaneous Pedicle Screw Fixation in Thoracolumbar Fracture

Clinics in Orthopedic Surgery • Vol. 11, No. 3, 2019• www.ecios.org

Table 2. Comparison of Radiological Findings between Groups A and B

\begin{tabular}{|c|c|c|c|c|}
\hline Radiologic finding & Group & Mean \pm SD & Median (range) & $p$-value \\
\hline \multirow[t]{2}{*}{ VAS } & A & $1.1 \pm 0.9$ & 1 (0 to 2$)$ & $>0.05$ \\
\hline & B & $1.3 \pm 1.2$ & 1 (0 to 3$)$ & \\
\hline \multirow[t]{2}{*}{ ODI } & A & $10.7 \pm 7.3$ & 10 (2 to 24$)$ & $>0.05$ \\
\hline & B & $9.2 \pm 7.1$ & 6 (4 to 21) & \\
\hline \multirow[t]{2}{*}{$\operatorname{ROM}\left(^{\circ}\right)$} & $A$ & $6.3 \pm 3.2$ & $3.7(1.2$ to 11.2$)$ & $<0.05$ \\
\hline & B & $1.3 \pm 0.9$ & 1 (0.1 to 2.4$)$ & \\
\hline \multirow[t]{2}{*}{ AVHR loss (\%) } & $A$ & $6.5 \pm 3.8$ & $6.4(2.1$ to 13.3$)$ & $>0.05$ \\
\hline & B & $3.1 \pm 3.6$ & 1.4 (0 to 8.6) & \\
\hline \multirow[t]{2}{*}{$\mathrm{CA}\left({ }^{\circ}\right)$} & $A$ & $4.1 \pm 13.1$ & 3.4 (-15.6 to 28.8) & $>0.05$ \\
\hline & B & $5.0 \pm 10.3$ & 5.9 (-15.8 to 20.8) & \\
\hline
\end{tabular}

Group A: early removal group (mean implant removal time, 8.5 months), Group B: late removal group (mean implant removal time, 16.1 months).

SD: standard deviation, VAS: visual analog scale, ODI: Oswestry disability index, ROM: range of motion, AVHR: anterior vertebral height ratio, CA: Cobb angle.

\section{Table 3. Changes in Clinical Scores between the Preoperative and Last Follow-up Period}

\begin{tabular}{crcr} 
Clinical score & Preoperative & Last follow-up & $p$-value \\
VAS & $6.7 \pm 1.1$ & $1.2 \pm 1.2$ & $<0.05$ \\
ODI & $35.9 \pm 7.3$ & $9.5 \pm 6.1$ & $<0.05$ \\
\hline
\end{tabular}

Values are presented as mean \pm standard deviation.

VAS: visual analog scale, ODI: Oswestry disability index.

graphs were obtained preoperatively, immediately postoperatively, and at each follow-up. AVHR was calculated as the percentage of $\mathrm{AVH}$ at the fracture level (AVH0) relative to the mean anterior height of the upper and lower adjacent vertebrae ([AVH1 / AVH2] / 2). CA was measured between the superior endplate of the upper vertebrae and the inferior endplate of the lower vertebrae. Intersegmental ROM was calculated by using CA at flexion and extension after implant removal in 16 patients. Preoperatively, flexion and extension radiographs were not obtained due to risk of neurologic aggravation. To check inter- and intraclass correlation coefficient of the measurements, two independent researchers (a full-time orthopedic spine specialist [HYS] and a senior resident [HSO]) reviewed all data. Each observer performed measurement three times 2 weeks apart, and the means of measured values were used as the final values.
Table 4. Changes in Radiological Findings

\begin{tabular}{lcc}
\multirow{2}{*}{\multicolumn{1}{c}{ Variable }} & \multicolumn{2}{c}{ Radiological finding } \\
\cline { 2 - 3 } & AVHR $(\%)$ & CA $\left({ }^{\circ}\right)^{*}$ \\
Preoperative & $71.9 \pm 11.2$ & $9.1 \pm 11.9$ \\
Immediate postoperative & $91.7 \pm 7.6$ & $0.8 \pm 14.7$ \\
After implant removal & $87.9 \pm 9.1$ & $2.7 \pm 13.7$ \\
Last follow-up & $86.6 \pm 9.4$ & $4.6 \pm 11.9$ \\
Total correction loss & $5.0 \pm 5.8$ & $3.9 \pm 7.3$ \\
\hline
\end{tabular}

Values are presented as mean \pm standard deviation. AVHR: anterior vertebral height ratio, CA: Cobb angle. ${ }^{*} p<0.05$.

\section{Statistical Analysis}

Data were analyzed using SPSS ver. 18.0 (SPSS Inc., Chicago, IL, USA). Paired $t$-test was used to determine the significance of intergroup differences in preoperative, postoperative, and last follow-up values (Tables 3 and 4) presented as mean and standard deviation. Mann-Whitney $U$-test was used to determine the significance of differences between group A and group B (Table 2) presented as median with maximum and minimum values. $\mathrm{A} p<0.05$ was considered significant. 
Oh and Seo. Percutaneous Pedicle Screw Fixation in Thoracolumbar Fracture

Clinics in Orthopedic Surgery • Vol. 11, No. 3, 2019• www.ecios.org

\section{RESULTS}

\section{Clinical Results}

Mean VAS score was 6.7 before surgery and 1.2 at final follow-up. Mean ODI score was 35.9 before surgery and 9.5 at final follow-up. At the final follow up, significant pain relief was achieved compared to preoperative values $(p<$ 0.05 ) (Table 3). There were no significant differences in VAS and ODI between group A and group B $(p>0.05)$.

\section{Radiological Results}

Postoperative AVHR and CA were significantly improved compared to preoperative values. Preoperative mean AVHR was $71.9 \%$, whereas postoperative mean AVHR was $91.7 \%(p<0.05)$. Immediately after implant removal and at the last follow-up, mean AVHR was $87.9 \%$ and $86.6 \%$, respectively, showing some correction loss at the last follow-up $(p<0.05)$. The mean CA decreased from $9.1^{\circ}$ preoperatively to $0.8^{\circ}$ postoperatively $(p<0.05)$. Despite the small increase in mean CA at the last follow-up to $4.6^{\circ}$, this value was not significantly higher than the mean postoperative CA (Table 4). Intersegmental ROM was calculated at the last follow-up with Cobb's method in 16 patients.
The mean ROM was $4.1^{\circ}$. It showed a slight improvement compared to that just before implant removal, which was considered to be motionless.

Of the 16 patients who were evaluated for ROM, nine patients had implant removal within 12 months (group A) and seven patients had removal after 12 months (group B). Mean ROM of group A assessed after implant removal was significantly higher than that of group $B$ (mean: group $\mathrm{A}, 1.3^{\circ}$ vs. group $\mathrm{B}, 6.3^{\circ}, p<0.05$; median: group $\mathrm{A}, 3.7^{\circ}$ vs. group $\mathrm{B}, 1^{\circ}, p<0.05$ ) (Table 2, Figs. 1 and $2)$. AVHR loss and CA showed no significant difference between the two groups $(p>0.05)$.

\section{Surgery-Related Complications}

Two cases of screw breakage were observed when implants were removed. However, they showed satisfactory outcomes at the last follow-up. There was no other complication such as nonunion or postoperative infection.

\section{DISCUSSION}

Minimum percutaneous methods of spinal fixation have been developed to avoid complications including soft tis-
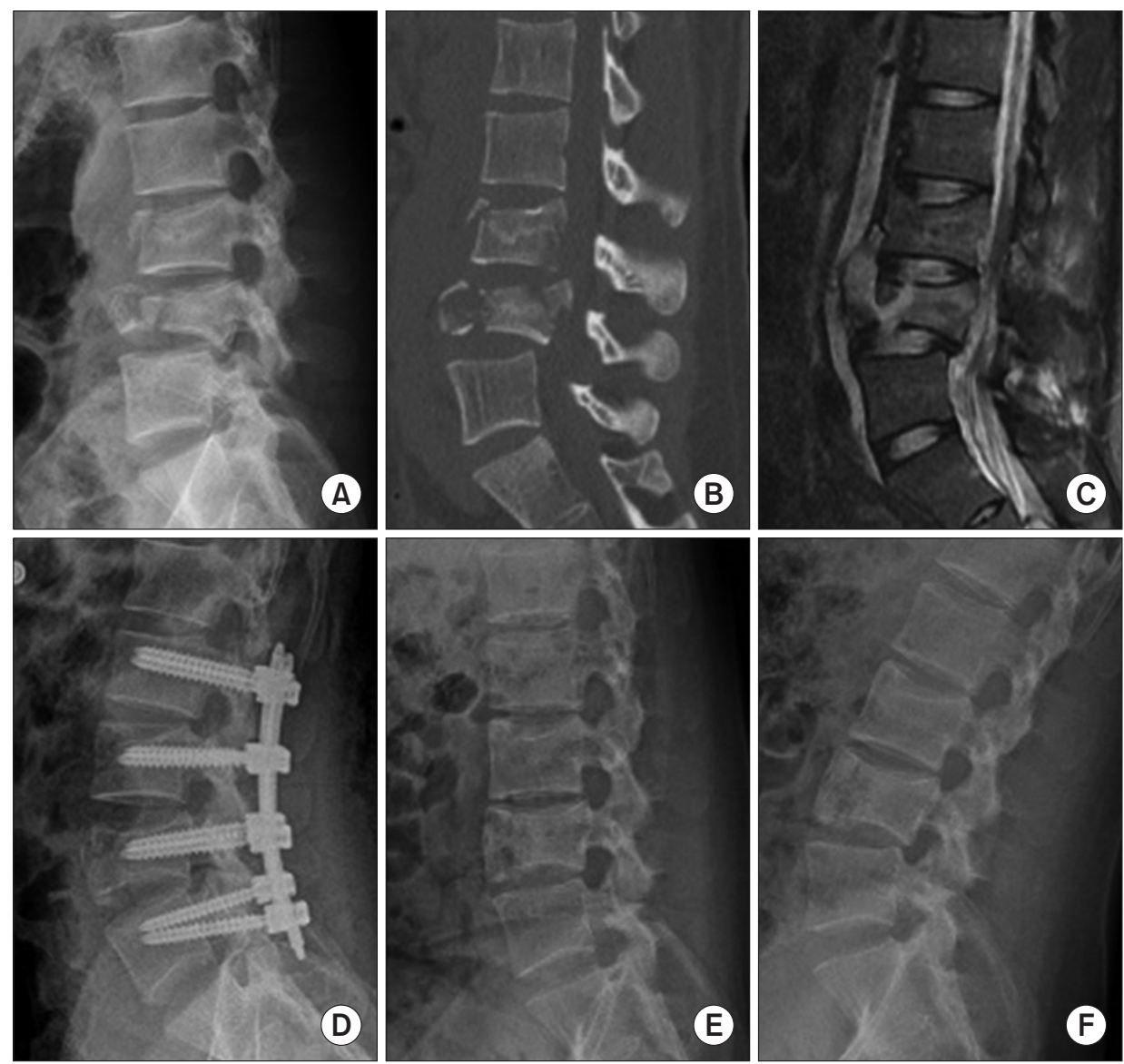

Fig. 1. A 24-year-old man with an $L 3-4$ burst fracture (group A: early removal group; mean implant removal time, 8.5 months). Preoperative plain radiograph (A) and computed tomography scan (B) showing $48 \%$ anterior vertebral height loss (L4) with posterior ligamentous complex injury (C). (D) Immediate postoperative plain radiograph showing restoration of anterior vertebral height ratio and Cobb angle. (E, F) The range of motion was $11.2^{\circ}$ on the flexionextension view after implant removal at 6 months after surgery. 

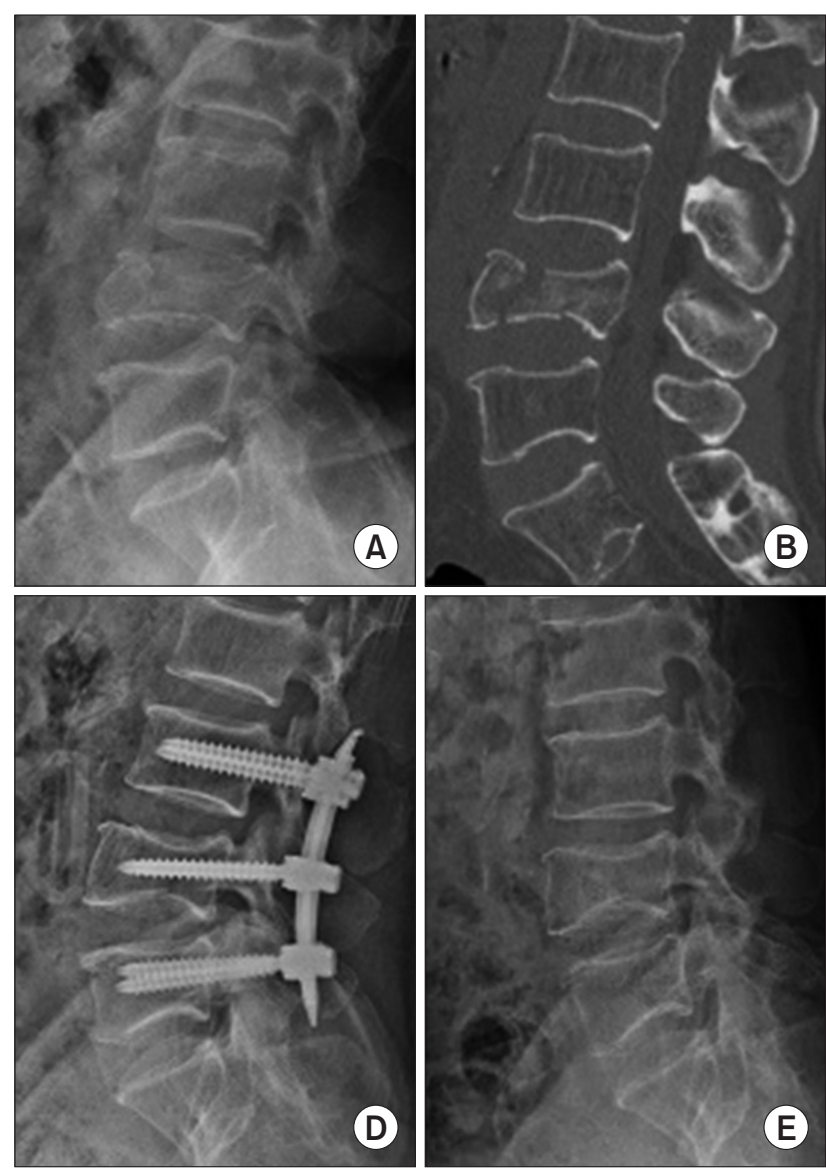

E

sue damage, muscular denervation, atrophy, and pain that occur in traditional spinal fixation. In many studies, compared with the conventional pedicle screw fixation, PPSF significantly reduced complications such as postoperative pain, surgical time, and bleeding, although the clinical, functional, and radiological outcomes of PPSF are almost the same as the conventional technique. ${ }^{4-6)}$ In addition, PPSF followed by implant removal after bone consolidation has been found to have advantages of early ambulation, minimal tissue injury, and complete motion reservation. Some studies have shown that after natural bone healing, implant removal makes it possible to preserve motion, leading to satisfactory outcomes. ${ }^{8,9)}$

In our study, sagittal deformity was improved after surgery in all patients. The correction was maintained with small losses of AVHR (preoperatively, 71.9\%; postoperatively, 91.7\%; and at last follow-up, 86.6\%) and CA (preoperatively, $9.1^{\circ}$; postoperatively, $0.8^{\circ}$; and at last follow-up, $4.6^{\circ}$ ) until the last follow-up after implant removal. It was difficult to determine the reason for the correction loss because we did not check MRI after surgery in our patients. However, in previous studies of kyphosis and vertebral collapse, intervertebral disc collapse was identified as the
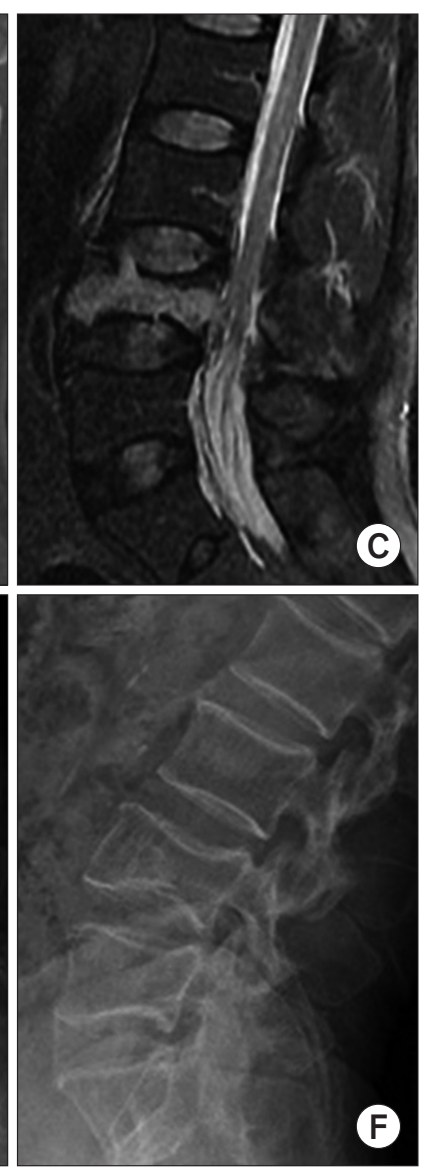

Fig. 2. A 64-year-old man with an $L 4$ burst fracture (group B: late removal group; mean implant removal time, 16.1 months). Preoperative plain radiograph (A) and computed tomography scan (B) showing 30\% anterior vertebral height loss with posterior ligamentous complex injury (C). (D) Immediate postoperative plain radiograph showing restoration of anterior vertebral height ratio and Cobb angle. ( $E, F)$ The range of motion was $0.6^{\circ}$ on the flexion-extension view after implant removal at 19 months after surgery.

main cause of postoperative kyphosis in patients with thoracolumbar fracture. In addition, disc creeping into the depression of the vertebra through central depression of the end plate can contribute to kyphosis and vertebral collapse. ${ }^{10-12)}$

Mean ROM after implant removal was $4.1^{\circ}$, meaning some restoration of ROM, but not as much as we had expected. Although it took a relatively short time (mean, 1 year) to remove the implant, it could be considered that the stiffness of the joint occurred and the ROM was not fully recovered. We divided patients into two groups according to the timing of implant removal to determine how the timing of removal affects the recovery of ROM and decide proper time for implant removal. Patients who had implant removal within 12 months after surgery had better ROM recovery than those who had implant removed after 12 months. There were no significant differences in AVHR loss and CA between the two groups. This suggests that consolidation progressed in most cases and stiffness was prevented in those with early implant removal within 12 months.

There were two cases of screw breakage in our study. We usually used 7.5-mm pedicle screws for PPSF. How- 
Oh and Seo. Percutaneous Pedicle Screw Fixation in Thoracolumbar Fracture

Clinics in Orthopedic Surgery • Vol. 11, No. 3, $2019 \bullet$ www.ecios.org

ever, for patients with narrow pedicles, we attempted to use 6.5 - $\mathrm{mm}$ screws. In the two cases of screw breakage, the screw diameter was $6.5 \mathrm{~mm}$, which might have affected screw breakage. In consideration of these cases, placing large pedicle screws into smaller-diameter pedicles seems reasonable and can prevent instrumentation failure. ${ }^{13)}$

This study has some limitations. First, this was a retrospective study reviewing collected clinical information of patients. There might have been selection bias. A prospective study involving subgroups divided by implant removal time is needed to decide that the exact timing of implant removal. In addition, the number of patients included in the study $(\mathrm{N}=30)$ and in ROM evaluation $(\mathrm{n}=16)$ was too small for optimal statistical analysis. Moreover, the mean follow-up period was relatively short: 18.3 months after surgery and 5.5 months after implant removal.

In summary, PPSF followed by implant removal after consolidation of vertebrae appears to be a safe and effective treatment that can provide restoration of ROM. It is necessary to establish the appropriate timing for metal removal between the time of joint stiffness and the time of bone union. In our study, early removal of implant within 12 months after surgery provided better achievement of ROM than removal after 12 months. However, there was no significant difference in AVHR between early removal and late removal groups.

\section{CONFLICT OF INTEREST}

No potential conflict of interest relevant to this article was reported.

\section{REFERENCES}

1. Fu Z, Zhang X, Shi Y, Dong Q. Comparison of surgical outcomes between short-segment open and percutaneous pedicle screw fixation techniques for thoracolumbar fractures. Med Sci Monit. 2016;22:3177-85.

2. McLain RF, Sparling E, Benson DR. Early failure of shortsegment pedicle instrumentation for thoracolumbar fractures: a preliminary report. J Bone Joint Surg Am. 1993;75(2):162-7.

3. Butt MF, Farooq M, Mir B, Dhar AS, Hussain A, Mumtaz M. Management of unstable thoracolumbar spinal injuries by posterior short segment spinal fixation. Int Orthop. 2007;31(2):259-64.

4. Li K, Li Z, Ren X, et al. Effect of the percutaneous pedicle screw fixation at the fractured vertebra on the treatment of thoracolumbar fractures. Int Orthop. 2016;40(6):1103-10.

5. Weber BR, Grob D, Dvorak J, Muntener M. Posterior surgical approach to the lumbar spine and its effect on the multifidus muscle. Spine (Phila Pa 1976). 1997;22(15):1765-72.

6. Kramer M, Katzmaier P, Eisele R, Ebert V, Kinzl L, Hartwig E. Surface electromyography-verified muscular damage associated with the open dorsal approach to the lumbar spine. Eur Spine J. 2001;10(5):414-20.

7. Grossbach AJ, Dahdaleh NS, Abel TJ, Woods GD, Dlouhy BJ, Hitchon PW. Flexion-distraction injuries of the thora- columbar spine: open fusion versus percutaneous pedicle screw fixation. Neurosurg Focus. 2013;35(2):E2.

8. Kim HS, Kim SW, Ju CI, Wang HS, Lee SM, Kim DM. Implant removal after percutaneous short segment fixation for thoracolumbar burst fracture: does it preserve motion? J Korean Neurosurg Soc. 2014;55(2):73-7.

9. Axelsson P, Stromqvist B. Can implant removal restore mobility after fracture of the thoracolumbar segment? Acta Orthop. 2016;87(5):511-5.

10. Chen JX, Xu DL, Sheng SR, et al. Risk factors of kyphosis recurrence after implant removal in thoracolumbar burst fractures following posterior short-segment fixation. Int Orthop. 2016;40(6):1253-60.

11. Alanay A, Acaroglu E, Yazici M, Oznur A, Surat A. Shortsegment pedicle instrumentation of thoracolumbar burst fractures: does transpedicular intracorporeal grafting prevent early failure? Spine (Phila Pa 1976). 2001;26(2):213-7.

12. Wang XY, Dai LY, Xu HZ, Chi YL. Kyphosis recurrence after posterior short-segment fixation in thoracolumbar burst fractures. J Neurosurg Spine. 2008;8(3):246-54.

13. Misenhimer GR, Peek RD, Wiltse LL, Rothman SL, Widell $\mathrm{EH}$ Jr. Anatomic analysis of pedicle cortical and cancellous diameter as related to screw size. Spine (Phila Pa 1976). 1989;14(4):367-72. 